\title{
Complex Partial Seizure as a Manifestation of Non-Ketotic Hyperglycemia: The Needle Recovered From Haystack?
}

\author{
Khairil Amir Rani ${ }^{a}$, Mohamed H. Ahmed ${ }^{a}$, , Louise Dunphy ${ }^{\mathrm{a}}$, Yousif Behnam ${ }^{\mathrm{a}}$
}

\begin{abstract}
We present a case of a 75-year-old gentleman with undiagnosed type 2 diabetes mellitus presenting with acute onset expressive dysphasia and right hemi-paresis with no prior history of seizure. He developed clusters of stereotypical complex partial seizures which were refractory to anti-epileptic agents. He was not known to have diabetes and his brain MRI was normal. His random blood sugar measurement on admission to hospital was $30 \mathrm{mmol} / \mathrm{L}$ with $\mathrm{HbA} 1 \mathrm{c}$ measurement of $14.8 \%$. His seizures terminated completely when his hyperglycemia was corrected with insulin and rehydration therapy.
\end{abstract}

Keywords: Non-ketotic hyperglycemia; Seizures; MRI

\section{Introduction}

First onset seizure in a patient aged over 50 years would normally suggest an intra-cranial lesion as an initial hypothesis. However, it could also be related to an underlying metabolic disturbance such as non-ketotic hyperglycemia (NKH) seen in diabetes (generally type 2). Rapid diagnosis is essential for seizure management, as they are usually refractory to anti-epileptic agents, stopping spontaneously when the hyperglycemia is corrected [1-3]. The global prevalence of diabetes has increased significantly. For instance, diabetes mellitus (DM) affects about $8.3 \%$ of the total world population and represents a challenge for all health authorities across the globe. It is estimated that 382 million people worldwide have diabetes, and this will increase to 592 million by 2035 . Importantly, it is estimated that as many as 175 million people with DM worldwide, or close to half of all people with DM, are unaware of their disease [4-6]. Therefore, in the era of diabetes epidemic, we believe it is crucial that acute and emergency medicine physicians should familiarize themselves with all diabetes complications, including neurological manifestations of NKH.

Manuscript accepted for publication April 04, 2016

aDepartment of Medicine, Milton Keynes University Hospital NHS Foundation Trust, Eaglestone, Milton Keynes, Buckinghamshire, UK

${ }^{b}$ Corresponding Author: Mohamed H. Ahmed, Department of Medicine, Milton Keynes University Hospital NHS Foundation Trust, Eaglestone, Milton Keynes, Buckinghamshire, UK. Email: elziber@yahoo.com

doi: http://dx.doi.org/10.14740/jocmr2552w

\section{Case Report}

A 75-year-old gentleman presented to the accident and emergency department (A\&E) following an episode of fall at home and was found by his wife on a bathroom floor. Initial neurological examination revealed global aphasia with right-sided hemiparesis. He was noted to be incontinent of urine.

His medical history included atrial fibrillation, hypertension, benign prostatic hypertrophy and chronic obstructive pulmonary disease. His medications included warfarin, tamsulosin, seretide, felodipine and candesartan. He has no physical disability, independent prior to hospital admission. On presentation, he was hemodynamically stable with a GCS of 11/15 (E4/V1/M6). Initial blood parameters including inflammatory markers were within normal limits. Each episode of seizures lasted less than a minute. They were all self-terminating. He was commenced on phenytoin infusion. CT brain revealed an old lacunar infarct in the left basal ganglia. He was transferred to the stroke ward for further observation and treatment. It was noted he had been developing further seizure clusters despite IV phenytoin. Each seizure episode lasted between 3 and 5

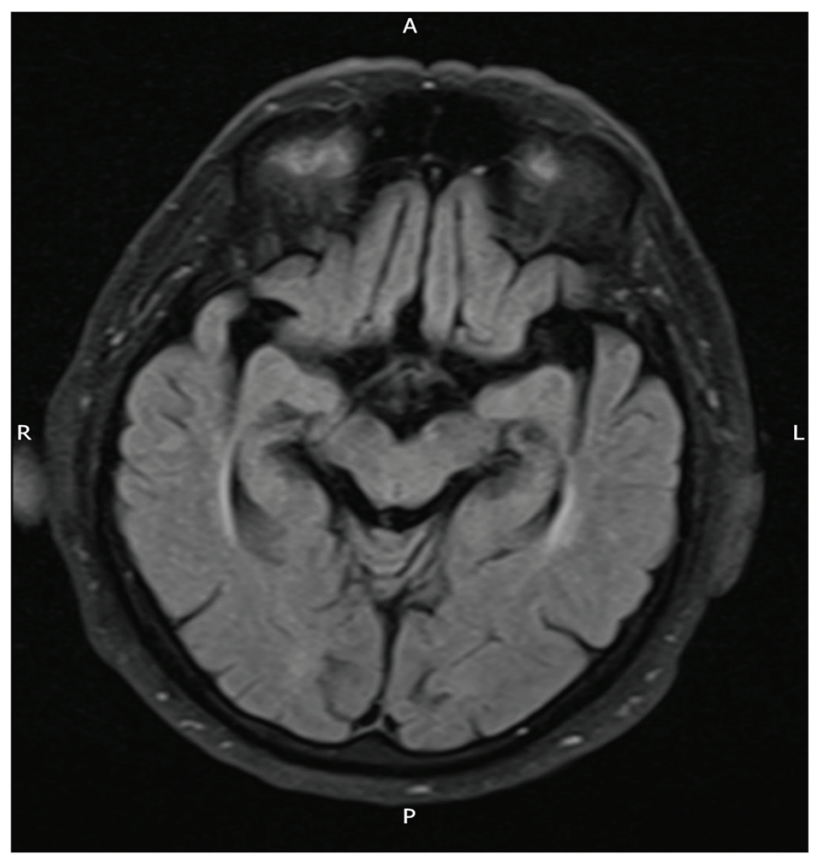

Figure 1. Normal MRI brain with no temporal or leptomeningeal enhancement (T2 FLAIR sequence). 
min. Nursing documentation revealed at least 20 stereotypical movements within the first $24 \mathrm{~h}$.

On the second day of his admission, his CRP had risen and he was commenced treatment for aspiration pneumonia. MRI brain was unremarkable with no evidence of temporal or meningeal enhancement (Fig. 1). Lumbar puncture was delayed in view of his abnormal INR. His random blood sugar measurement was recorded at $30 \mathrm{mmol} / \mathrm{L}$ with $\mathrm{HbA} 1 \mathrm{c}$ measurement of $14.8 \%$. He was commenced on IV insulin with subsequent reduction of seizure frequency. He was seizure free within 24 $\mathrm{h}$ and discharged uneventfully with oral metformin. He was reportedly still seizure free on annual review without any antiepileptic medication.

\section{Discussion}

NKH is a clinical syndrome consisting of severe hyperglycemia, hyper-osmolarity and intra-cellular dehydration without keto-acidosis. Maccario et al first described focal seizures as a manifestation of hyperglycemia without keto-acidosis, in their report of seven cases, published in 1965 [1]. In the intervening years, several case series and retrospective reviews have described such a neuro-endocrine entity $[2,3]$. In majority of cases, seizures reveal previously undiagnosed diabetes and they may be preceded by osmotic symptoms [4].

Neurological deficits associated with NKH vary and can include an alteration in consciousness level. Motor seizures are most often partial and may be followed by a post-ictal motor deficit. According to the literature, they often begin as partial seizures that generalize secondarily [7]. Other presentations include visual hallucinations, aphasia, visual field defect and hemisensory loss $[8,9]$. States of "petit mal" are seen in the form of epilepsia partialis continua [10].

Pathophysiology for neurological manifestation of $\mathrm{NKH}$ remains unclear. A multi-factorial etiology has been suggested, with hyperglycemia as one of the factors likely to promote the onset of seizures. In 1973, Sing and Strobos suggested that hyperglycemia may create a hyper-osmolar gradient between the intra- and extra-cellular neuronal environments, thereby inducing intra-cellular dehydration, which induces seizures [11]. According to Ozer et al, hyperglycemia increases GABA metabolism and thereby diminishes the seizure threshold [12]. Dong Wook et al suggest reversible blood brain barrier (BBB) disruption as a cause of hyperglycemia-induced seizures in NKH patients [13]. However, Maccario concluded that serum hyper-osmolality, leading to intra-cellular dehydration and disturbance of electrolyte equilibrium of the intra-cellular and extra-cellular compartments, is the probable etiologic factor [14].

Correction of hyperglycemia, hyper-osmolarity or electrolyte disorders is vital. Initiation of insulin therapy and rehydration are required as correction of hyperglycemia stops seizures effectively. Anti-epileptic treatment is likely to aggravate seizures. Indeed, phenytoin inhibits insulin secretion and consequently is harmful.

This case report highlights the importance of a thorough clinical evaluation in patients presenting with stroke mimics. All patients presenting to the emergency department with stroke-like symptoms should have their blood sugar measured on arrival to avoid an unnecessary delay in initiating appropriate treatment.

\section{References}

1. Maccario M, Messis CP, Vastola EF. Focal Seizures as a Manifestation of Hyperglycemia without Ketoacidosis. A Report of Seven Cases with Review of the Literature. Neurology. 1965;15:195-206.

2. Brick JF, Gutrecht JA, Ringel RA. Reflex epilepsy and nonketotic hyperglycemia in the elderly: a specific neuroendocrine syndrome. Neurology. 1989;39(3):394-399.

3. Hennis A, Corbin D, Fraser H. Focal seizures and nonketotic hyperglycaemia. J Neurol Neurosurg Psychiatry. 1992;55(3):195-197.

4. Danaei G, Finucane MM, Lu Y, Singh GM, Cowan MJ, Paciorek CJ, Lin JK, et al. National, regional, and global trends in fasting plasma glucose and diabetes prevalence since 1980: systematic analysis of health examination surveys and epidemiological studies with 370 country-years and 2.7 million participants. Lancet. 2011;378(9785):3140.

5. Nandeshwar S, Jamara V, Pal D. Indian diabetes risk score for screening of undiagnosed diabetic subjects of Bhopal city. National Journal of Community Medicine. 2010;1:176-177.

6. International Diabetes Federation. IDF Diabetes Atlas, 6th edn. Brussels, Belgium: International Diabetes Federation, 2013. http://www.idf.org/diabetesatlas.

7. Tiamkao S, Pratipanawatr T, Nitinavakarn B, Chotmongkol V, Jitpimolmard S. Seizures in nonketotic hyperglycaemia. Seizure. 2003;12(6):409-410.

8. Wegierko J. [Typical syndrome of clinical manifestations in diabetes mellitus with fatal termination in coma without ketonic acidemia; so-called third coma]. Pol Tyg Lek (Wars). 1956;11(48):2020-2023.

9. Pavel I, Bonaparte H, Pirvulescu M, Covanov D. [Hyperosmotic Diabetic Coma without Acido-Ketosis]. Stud Cercet Med Interna. 1965;40:145-155.

10. Cochin JP, Hannequin D, Delangre T, Guegan-Massardier E, Augustin P. [Continuous partial epilepsy disclosing diabetes mellitus]. Rev Neurol (Paris). 1994;150(3):239241.

11. Singh BM, Strobos RJ. Epilepsia partialis continua associated with nonketotic hyperglycemia: clinical and biochemical profile of 21 patients. Ann Neurol. 1980;8(2):155-160.

12. Ozer F, Mutlu A, Ozkayran T. Reflex epilepsy and nonketotic hyperglycemia. Epileptic Disord. 2003;5(3):165168.

13. Dong Wook K, Yeonsil M, Hong G, Jin Woo C, Jeeyoung O. Blood-brain barrier disruption is Involves in Seizure and Hemianopia in Non-ketotic Hyperglycaemia. The Neurologist. 2011;17(3).

14. Maccario M. Neurological dysfunction associated with nonketotic hyperglycemia. Arch Neurol. 1968;19(5):525534. 